ITM Web of Conferences 4, 01004 (2015)

DOI: $10.1051 /$ itmconf/20150401004

(C) Owned by the authors, published by EDP Sciences, 2015

\title{
Mathematical model for pulsed chemotherapy
}

\author{
Ahmed Lakmeche ${ }^{\mathrm{a}}$, Mohamed Helal ${ }^{\mathrm{b}}$, and Abdelkader Lakmeche ${ }^{\mathrm{c}}$ \\ Laboratory of Biomathematics, Univ. Sidi Bel Abbes, PB. 89, Sidi Bel Abbes 22000, Algeria
}

\begin{abstract}
A pulsed chemotherapeutic treatment model is investigated in this work. We prove the existence of nontrivial periodic solutions by the mean of Lyapunov-Schmidt bifurcation method of a cancer model. In this model we consider the case of application of two drugs, the first one $\mathbf{P}$ with continuous effect, it appears in the differential equations, and the second one $\mathbf{T}$ with instantaneous effects expressed by impulse equations. The existence of bifurcated nontrivial periodic solutions are discussed with respect to the competition parameter values.
\end{abstract}

\section{Introduction}

In this work a mathematical model for cancer chemotherapy is studied by considering interactions between tumor and normal cells. This model consists of three nonlinear ordinary differential equations describing the dynamic of the cancer under the continuous effect of a drug $\mathbf{P}$, and three discrete equations describing the instantaneous effects of a drug $\mathbf{T}$ on the different types of cancerous cells, it is called pulsed-therapy. When the drug $\mathbf{P}$ is used, a fraction of normal and sensitive cells are killed, the effect of $\mathbf{P}$ is expressed by the rates $p_{1}$ and $p_{2}$ in the differential equations describing the dynamics of normal and sensitive cells. The drug $\mathbf{T}$ has an instantaneous effect described by impulse effects on all kinds of cells. The model studied in this work is a generalization of those considered in [1, 5-7] and [10], where only one drug with impulse effects is considered. We use similar approach to that used in [7] to find conditions of stability of trivial solution and bifurcation of nontrivial periodic solutions corresponding to eradication of the tumor and its persistence, respectively. Some recent works have considered models similar to our model, see for instance $[3,5,8]$ and [11].

Our work is organized as follow, in the next two sections we define variables and parameters of the model and the approach of fixed point. In section four we study the stability of trivial solution and the bifurcation of periodic nontrivial solutions. In the last section we give some concluding remarks.

\footnotetext{
a e-mail: lakahmed2000@yahoo.fr

b e-mail: mhelal_abbes@yahoo.fr

c e-mail: lakmeche@yahoo.fr
}

This is an Open Access article distributed under the terms of the Creative Commons Attribution License 4.0, which permits unrestricted use, distribution, and reproduction in any medium, provided the original work is properly cited. 


\section{The model}

The model studied in this work is the following

$$
\begin{gathered}
\dot{x}_{1}(t)=r_{1} x_{1}\left(1-\frac{x_{1}}{K_{1}}-\lambda_{1}\left(x_{2}+x_{3}\right)\right)-p_{1} x_{1}, \\
\dot{x}_{2}(t)=r_{2} x_{2}\left(1-\frac{x_{2}+x_{3}}{K_{2}}-\lambda_{2}\left(x_{1}+x_{3}\right)\right)-m x_{2}-p_{2} x_{2}, \\
\dot{x}_{3}(t)=r_{3} x_{3}\left(1-\frac{x_{2}+x_{3}}{K_{3}}-\lambda_{3}\left(x_{1}+x_{2}\right)\right)+m x_{2}, \\
x_{1}\left(t_{i}^{+}\right)=T_{1} x_{1}\left(t_{i}\right) \\
x_{2}\left(t_{i}^{+}\right)=\left(T_{2}-R\right) x_{2}\left(t_{i}\right), \\
x_{3}\left(t_{i}^{+}\right)=T_{3} x_{3}\left(t_{i}\right)+R x_{2}\left(t_{i}\right),
\end{gathered}
$$

where $t_{i+1}-t_{i}=\tau>0, \forall i \in \mathbb{N}$, and for $j=\overline{1,3}, T_{j}, R$ are positive constants.

The variables and parameters are

$\tau$ : period between two successive $\mathbf{T}$ drug treatment,

$x_{j}$ : normal (resp. sensitive tumor and resistant tumor) cell biomass for $j=1$ (resp. 2,3),

$r_{j}$ : growth rate of the normal (resp. sensitive tumor and resistant tumor) cells for $j=1$ (resp. 2,3),

$k_{j}$ : carrying capacity of the normal (resp. sensitive tumor and resistant tumor) cells for $j=1$ (resp. 2,3),

$\lambda_{j}$ : competitive parameter of the normal (resp. sensitive tumor and resistant tumor) cells for $j=1$ (resp. 2,3),

$T_{j}$ : survival fraction of the normal (resp. sensitive tumor and resistant tumor) cells for $j=1$ (resp. 2,3), their values are completely determined by the quantity of injected $\mathbf{T}$ drug dose,

$R$ : fraction of cells mutating due to the dose of the $\mathbf{T}$ drug which is less than $T_{2}$,

$m$ : acquired resistance parameter usually it is very small (see [9]),

$p_{1}$ (resp. $p_{2}$ ): death rate fraction of the normal (resp. sensitive tumor) cells, due to the drug $\mathbf{P}$ which is less than $r_{1}$ (resp. $r_{2}-m$ ).

\section{The fixed point approach}

We study bifurcation of nontrivial periodic solution, which occurs at $e_{0}^{\prime}=0 \neq i_{0}^{\prime}$ or $e_{0}^{\prime} \neq 0=i_{0}^{\prime}$. To this purpose, we shall employ a fixed point argument.

Let $\Phi\left(t, X_{0}\right)=\left(\Phi_{1}\left(t, X_{0}\right), \Phi_{2}\left(t, X_{0}\right), \Phi_{3}\left(t, X_{0}\right)\right)$ the solution of the system (1)-(3) for the initial condition $X_{0}=\zeta(0,0,0)$. We assume that the flow $\Phi$ applies up to time $\tau$. So, $\xi(\tau)=\Phi\left(\tau, X_{0}\right)$. Then, within a very small time interval starting at time $\tau$, we assume that the treatment $\mathbf{T}$ is administered and kills instantaneously a fraction of the population.

We denote by $\Theta: \mathbb{R}^{3} \rightarrow \mathbb{R}^{3}$ and $F: \mathbb{R}^{3} \rightarrow \mathbb{R}^{3}$ the maps defined by

$$
\begin{aligned}
\Theta\left(x_{1}, x_{2}, x_{3}\right) & =\left(\Theta_{1}\left(x_{1}, x_{2}, x_{3}\right), \Theta_{2}\left(x_{1}, x_{2}, x_{3}\right), \Theta_{3}\left(x_{1}, x_{2}, x_{3}\right)\right) \\
& =\left(T_{1} x_{1},\left(T_{2}-R\right) x_{2}, T_{3} x_{3}+R x_{2}\right), \\
F\left(x_{1}, x_{2}, x_{3}\right) & =\left(F_{1}\left(x_{1}, x_{2}, x_{3}\right), F_{2}\left(x_{1}, x_{2}, x_{3}\right), F_{3}\left(x_{1}, x_{2}, x_{3}\right)\right),
\end{aligned}
$$




$$
\begin{gathered}
F_{1}\left(x_{1}, x_{2}, x_{3}\right)=r_{1} x_{1}\left(1-\frac{x_{1}}{K_{1}}-\lambda_{1}\left(x_{2}+x_{3}\right)\right)-p_{1} x_{1}, \\
F_{2}\left(x_{1}, x_{2}, x_{3}\right)=r_{2} x_{2}\left(1-\frac{x_{2}+x_{3}}{K_{2}}-\lambda_{2}\left(x_{1}+x_{3}\right)\right)-m x_{2}-p_{2} x_{2}
\end{gathered}
$$

and

$$
F_{3}\left(x_{1}, x_{2}, x_{3}\right)=r_{3} x_{3}\left(1-\frac{x_{2}+x_{3}}{K_{3}}-\lambda_{3}\left(x_{1}+x_{2}\right)\right)+m x_{2} .
$$

Let the operator be $\Psi: \mathbb{R}^{3} \rightarrow \mathbb{R}^{3}$ defined by

$$
\Psi\left(\tau, X_{0}\right)=\left(\Psi_{1}\left(\tau, X_{0}\right), \Psi_{2}\left(\tau, X_{0}\right), \Psi_{3}\left(\tau, X_{0}\right)\right)=\Theta\left(\Phi\left(\tau, X_{0}\right)\right) .
$$

The term $\xi\left(\tau^{+}\right)$denotes the state of the population after the use of $\mathbf{T}$ treatment, $\xi\left(\tau^{+}\right)$is determined in terms of $\xi(\tau)$ according to Eqs. (4)-(6). We have $\xi\left(\tau^{+}\right)=\Theta(\xi(\tau))=\Theta\left(\Phi\left(\tau, X_{0}\right)\right)$.

We reduce the problem of finding a periodic solution of (1)-(6) to a fixed point problem. Then $\xi=\Phi\left(., X_{0}\right)$ is a $\tau$-periodic solution of (1)-(6) if and only if its initial condition $X_{0}$ is a fixed point for $\Psi=\Psi\left(., X_{0}\right)$.

A fixed point $X_{0}$ of $\Psi(\tau,$.$) is the initial state of (1)-(6) which gives a \tau$-periodic solution $\xi$ verifying $\xi(0)=X_{0}$. Consequently, to establish the existence of nontrivial periodic solutions of (1)-(6), we need to prove the existence of nontrivial fixed points of $\Psi$ by the mean of bifurcation analysis.

When the cancer cells are eradicated $\left(x_{2}=x_{3}=0\right)$, then Eqs. (1)-(6) are reduced to the subsystem

$$
\begin{gathered}
\dot{x}_{1}(t)=r_{1} x_{1}\left(1-\frac{x_{1}}{K_{1}}-\right)-p_{1} x_{1}, \quad 0<t \leq \tau_{0}, \\
x_{1}\left(t_{i}^{+}\right)=T_{1} x_{1}\left(t_{i}\right)
\end{gathered}
$$

where $\tau_{0} \in \mathbb{R}_{+}$and $t_{i}=i \tau_{0}, i \in \mathbb{N}^{*}$.

Theorem 3.1: If the condition $\tau_{0}>\frac{\ln \left(\frac{1}{T_{1}}\right)}{r_{1}-p_{1}}$ is satisfied, then the trivial periodic solution $x_{s}$ of (7)-(8) is exponentially stable.

Proof: It's easy to show that (7)-(8) has a $\tau_{0}$-periodic solution $x\left(t, x_{0}\right)=x_{s}(t)$, where

$$
x_{s}(t)=\frac{k_{1}\left(\frac{r_{1}-p_{1}}{r_{1}}\right)\left(T_{1}-\exp \left(-\left(r_{1}-p_{1}\right) \tau_{0}\right)\right) \exp \left(\left(r_{1}-p_{1}\right) t\right)}{\exp \left(\left(r_{1}-p_{1}\right) t\right)\left(T_{1}-\exp \left(-\left(r_{1}-p_{1}\right) \tau_{0}\right)\right)+\left(1-T_{1}\right)}, \quad 0<t \leq \tau_{0},
$$

with $x_{0}=\frac{k_{1}\left(r_{1}-p_{1}\right)\left(T_{1}-\exp \left(-\left(r_{1}-p_{1}\right) \tau_{0}\right)\right)}{r_{1}\left(1-\exp \left(-\left(r_{1}-p_{1}\right) \tau_{0}\right)\right)}$.

The solution given by (9) is defined and stable in the one dimensional space if $T_{1}>\exp \left(-\left(r_{1}-p_{1}\right) \tau_{0}\right)$. That is

$$
\tau_{0}>\frac{\ln \left(\frac{1}{T_{1}}\right)}{\left(r_{1}-p_{1}\right)}
$$

The function $\zeta=\left(x_{s}, 0,0\right)$ is a periodic solution of (1)-(6), it's called a trivial periodic solution and its initial condition $\zeta_{0}=\zeta(0)=\left(x_{s}(0), 0,0\right)=\left(x_{0}, 0,0\right)$ is a fixed point of $\Psi\left(., X_{0}\right)$. 


\subsection{Stability of the trivial fixed point}

We have the following results.

Theorem 3.2: The trivial periodic solution $\zeta$ of (1)-(6) is exponentially stable if and only if

$$
\begin{gathered}
T_{2}<T_{1}^{\frac{r_{2} \lambda_{2} K_{1}}{r_{1}}}+R, \\
T_{3}<T_{1}^{\frac{r_{3} \lambda_{3} K_{1}}{r_{1}}}
\end{gathered}
$$

and

$$
\frac{\ln \left(\frac{1}{T_{1}}\right)}{r_{1}-p_{1}}<\tau_{0}<\min \left(\frac{\ln \left(T_{1}^{\frac{r_{2} \lambda_{2} K_{1}}{r_{1}}}\left(T_{2}-R\right)^{-1}\right)}{r_{2}\left(1-\lambda_{2} k_{1}+\frac{\lambda_{2} k_{1} p_{1}}{r_{1}}\right)-p_{2}-m}, \frac{\ln \left(T_{1}^{\frac{r_{3} \lambda_{3} K_{1}}{r_{1}}} T_{3}^{-1}\right)}{r\left(\left[1-\lambda_{3} k_{1}+\frac{\lambda_{3} k_{1} p_{1}}{r_{1}}\right)\right.}\right) .
$$

Proof: We have $D_{X} \Psi\left(\tau_{0}, X\right)=D_{X} \Theta\left(\Phi\left(\tau_{0}, X\right)\right) \frac{\partial \Phi}{\partial X}\left(\tau_{0}, X\right)$, then for $\zeta_{0}=\left(x_{0}, 0,0\right)$ we obtain

$$
\begin{aligned}
D_{X} \Psi\left(\tau_{0}, \zeta_{0}\right) & =D_{X} \Theta\left(\Phi\left(\tau_{0}, \zeta_{0}\right)\right) \frac{\partial \Phi}{\partial X}\left(\tau_{0}, \zeta_{0}\right) \\
& =\left(\begin{array}{ccc}
\frac{\partial \Theta_{1}\left(\Phi\left(\tau_{0}, \zeta_{0}\right)\right)}{\partial x_{1}} \frac{\partial \Phi_{1}\left(\tau_{0}, \zeta_{0}\right)}{\partial x_{1}} & \frac{\partial \Theta_{1}\left(\Phi\left(\tau_{0}, \zeta_{0}\right)\right)}{\partial x_{1}} \frac{\partial \Phi_{1}\left(\tau_{0}, \zeta_{0}\right)}{\partial x_{2}} \frac{\partial \Theta_{1}\left(\Phi\left(\tau_{0}, \zeta_{0}\right)\right)}{\partial x_{1}} \frac{\partial \Phi_{1}\left(\tau_{0}, \zeta_{0}\right)}{\partial x_{3}} \\
0 & \frac{\partial \Theta_{2}\left(\Phi\left(\tau_{0}, \zeta_{0}\right)\right)}{\partial x_{2}} \frac{\partial \Phi_{2}\left(\tau_{0}, \zeta_{0}\right)}{\partial x_{2}} & 0 \\
0 & * & \frac{\partial \Theta_{3}\left(\Phi\left(\tau_{0}, \zeta_{0}\right)\right)}{\partial x_{3}} \frac{\partial \Phi_{3}\left(\tau_{0}, \zeta_{0}\right)}{\partial x_{3}}
\end{array}\right)
\end{aligned}
$$

where

$$
\begin{aligned}
& *=\frac{\partial \Theta_{3}\left(\Phi\left(\tau_{0}, \zeta_{0}\right)\right)}{\partial x_{2}} \frac{\partial \Phi_{2}\left(\tau_{0}, \zeta_{0}\right)}{\partial x_{2}}+\frac{\partial \Theta_{3}\left(\Phi\left(\tau_{0}, \zeta_{0}\right)\right)}{\partial x_{3}} \frac{\partial \Phi_{3}\left(\tau_{0}, \zeta_{0}\right)}{\partial x_{2}}, \\
& \frac{\partial \Phi_{1}\left(\tau_{0}, \zeta_{0}\right)}{\partial x_{1}}=e^{-\left(r_{1}-p_{1}\right) \tau_{0}} T_{1}^{-2} \\
& \frac{\partial \Phi_{2}\left(\tau_{0}, \zeta_{0}\right)}{\partial x_{2}}=e^{\left(r_{2}-p_{2}-m\right) \tau_{0}} e^{-\frac{r_{2} \lambda_{2} k_{1}\left(r_{1}-p_{1}\right) \tau_{0}}{r_{1}}} T_{1}^{-\frac{r_{2} \lambda_{2} k_{1}}{r_{1}}}, \\
& \frac{\partial \Phi_{3}\left(\tau_{0}, \zeta_{0}\right)}{\partial x_{3}}=e^{r_{3} \tau_{0}} e^{-\frac{r_{3} \lambda_{3} k_{1}\left(r_{1}-p_{1}\right) \tau_{0}}{r_{1}}} T_{1}^{-\frac{r_{3} \lambda_{3} k_{1}}{r_{1}}}, \\
& \frac{\partial \Phi_{3}\left(\tau_{0}, X_{0}\right)}{\partial x_{2}}=\frac{m e^{r_{3} \tau_{0}} e^{-\frac{r_{3} \lambda_{3} k_{1}\left(r_{1}-p_{1}\right)}{r_{1}}} \tau_{0}}{\left(1-e^{-\left(r_{1}-p_{1}\right) \tau_{0}}\right)^{-\frac{\left(r_{2} \lambda_{2}-r_{3} \lambda_{3}\right) k_{1}}{r_{1}}}} \int_{0}^{-\frac{r_{3} \lambda_{3} k_{1}}{r_{1}}} \frac{e^{\left(r_{2}-p_{2}-m\right) u} I_{2}(u)}{e^{r_{3} u} I_{3}(u)} d u, \\
& \frac{\partial \Phi_{1}\left(\tau_{0}, X_{0}\right)}{\partial x_{2}}=-\frac{\lambda_{1} k_{1} e^{-\left(r_{1}-p_{1}\right) \tau_{0}} T_{1}^{-2}\left(r_{1}-p_{1}\right)\left(T_{1}-e^{-\left(r_{1}-p_{1}\right) \tau_{0}}\right)}{\left(1-e^{-\left(r_{1}-p_{1}\right) \tau_{0}}\right)^{2-\frac{r_{2} \lambda_{2} k_{1}}{r_{1}}}} \int_{0}^{\tau_{0}} e^{\left(r_{2}-p_{2}-m\right) u} I_{2}(u) d u \\
& -\frac{m \lambda_{1} k_{1} e^{-\left(r_{1}-p_{1}\right) \tau_{0}}\left(r_{1}-p_{1}\right)\left(T_{1}-e^{-\left(r_{1}-p_{1}\right) \tau_{0}}\right)}{T_{1}^{2}\left(1-e^{-\left(r_{1}-p_{1}\right) \tau_{0}}\right)^{2-\frac{r_{2} \lambda_{2} k_{1}}{r_{1}}}} \int_{0}^{\tau_{0}} e^{r_{3} s} I_{3}(s)\left(\int_{0}^{s} \frac{e^{\left(r_{2}-p_{2}-m\right) u} I_{2}(u)}{e^{r_{3} u} I_{3}(u)} d u\right) d s,
\end{aligned}
$$




$$
\begin{gathered}
\frac{\partial \Phi_{1}\left(\tau_{0}, X_{0}\right)}{\partial x_{3}}=-\frac{\lambda_{1} e^{-\left(r_{1}-p_{1}\right) \tau_{0}} k_{1}\left(r_{1}-p_{1}\right)\left(T_{1}-e^{-\left(r_{1}-p_{1}\right) \tau_{0}}\right)}{T_{1}^{2}\left(1-e^{-\left(r_{1}-p_{1}\right) \tau_{0}}\right)^{2-\frac{r_{3} \lambda_{3} k_{1}}{r_{1}}}} \int_{0}^{\tau_{0}} e^{r_{3} u} I_{3}(u) d u, \\
I_{2}(s)=\left(e^{\left(r_{1}-p_{1}\right) s}\left(T_{1}-e^{-\left(r_{1}-p_{1}\right) \tau_{0}}\right)+1-T_{1}\right)^{1-\frac{r_{2} \lambda_{2} k_{1}}{r_{1}}},
\end{gathered}
$$

and

$$
I_{3}(s)=\left(e^{\left(r_{1}-p_{1}\right) s}\left(T_{1}-e^{-\left(r_{1}-p_{1}\right) \tau_{0}}\right)+1-T_{1}\right)^{1-\frac{r_{3} \lambda_{3} k_{1}}{r_{1}}} .
$$

The solution $\zeta$ is exponentially stable if and only if the spectral radius of $D_{X} \Psi\left(\tau_{0}, \zeta_{0}\right)$ is less than one, that is

$$
\begin{gathered}
\tau_{0}>\frac{\ln \left(\frac{1}{T_{1}}\right)}{r_{1}-p_{1}}, \\
\left(T_{2}-R\right) e^{\left[r_{2}\left(1-\frac{\lambda_{2} k_{1}\left(r_{1}-p_{1}\right)}{r_{1}}\right)-p_{2}-m\right] \tau_{0}} T_{1}^{-\frac{r_{2} \lambda_{2} k_{1}}{r_{1}}}<1
\end{gathered}
$$

and

$$
T_{3} e^{\left[1-\frac{\lambda_{3} k_{1}\left(r_{1}-p_{1}\right)}{r_{1}}\right] r_{3} \tau_{0}} T_{1}^{-\frac{r_{3} \lambda_{3} k_{1}}{r_{1}}}<1 .
$$

In view of the fact that $\lambda_{2} K_{1}<1$ and $\lambda_{3} K_{1}<1$ (see [10]), we have

$$
T_{2}<T_{1}^{\frac{r_{2} \lambda_{2} K_{1}}{r_{1}}}+R
$$

and

$$
T_{3}<T_{1}^{\frac{r_{3} \lambda_{3} K_{1}}{r_{1}}} .
$$

Then $\zeta$ is stable as an equilibrium for the full system (1)-(6) if and only if

$$
\frac{\ln \left(\frac{1}{T_{1}}\right)}{r_{1}-p_{1}}<\tau_{0}<\min \left(\frac{\ln \left(T_{1}^{\frac{r_{2} \lambda_{2} K_{1}}{r_{1}}}\left(T_{2}-R\right)^{-1}\right)}{r_{2}\left(1-\lambda_{2} k_{1}+\frac{\lambda_{2} k_{1} p_{1}}{r_{1}}\right)-p_{2}-m}, \frac{\ln \left(T_{1}^{\frac{r_{3} \lambda_{3} K_{1}}{r_{1}}} T_{3}^{-1}\right)}{\left(1-\lambda_{3} k_{1}+\frac{\lambda_{3} k_{1} p_{1}}{r_{1}}\right)}\right) .
$$

\section{Critical cases}

In this section, we are interested in the bifurcation of nontrivial periodic solutions from $\zeta=\left(x_{S}, 0,0\right)$. To find a nontrivial periodic solution of period $\tau$ with initial condition $X$, we need to solve the fixed point problem

$$
X=\Psi(\tau, X) .
$$

Let $\bar{\tau}$ and $\bar{X}$ such that $\tau=\tau_{0}+\bar{\tau}$ and $X=X_{0}+\bar{X}$. The Eq. (16) is equivalent to

$$
M(\bar{\tau}, \bar{X})=0,
$$

where $M(\bar{\tau}, \bar{X})=\left(M_{1}(\bar{\tau}, \bar{X}), M_{2}(\bar{\tau}, \bar{X}), M_{3}(\bar{\tau}, \bar{X})\right)=X_{0}+\bar{X}-\Psi\left(\tau_{0}+\bar{\tau}, X_{0}+\bar{X}\right)$.

Since $\zeta$ is a trivial $\tau_{0}$-periodic solution of (1)-(6), then it is associated to the trivial fixed point $\zeta_{0}$ of $\Psi\left(\tau_{0},.\right)$. 
Let

$$
D_{X} M(0,0)=\left(\begin{array}{ccc}
a_{0}^{\prime} & b_{0}^{\prime} & c_{0}^{\prime} \\
0 & e_{0}^{\prime} & 0 \\
0 & h_{0}^{\prime} & i_{0}^{\prime}
\end{array}\right),
$$

where

$$
\begin{aligned}
& a_{0}^{\prime}=1-e^{-\left(r_{1}-p_{1}\right) \tau_{0}} T_{1}^{-1}, \\
& b_{0}^{\prime}=\frac{\lambda_{1} k_{1} e^{-\left(r_{1}-p_{1}\right) \tau_{0}} T_{1}^{-1}\left(r_{1}-p_{1}\right)\left(T_{1}-e^{-\left(r_{1}-p_{1}\right) \tau_{0}}\right)}{\left(1-e^{-\left(r_{1}-p_{1}\right) \tau_{0}}\right)^{2-\frac{r_{2} \lambda_{2} k_{1}}{r_{1}}}} \int_{0}^{\tau_{0}} e^{\left(r_{2}-p_{2}-m\right) u} I_{2}(u) d u \\
& +\frac{m \lambda_{1} k_{1} e^{-\left(r_{1}-p_{1}\right) \tau_{0}} T_{1}^{-1}\left(r_{1}-p_{1}\right)\left(T_{1}-e^{-\left(r_{1}-p_{1}\right) \tau_{0}}\right)}{\left(1-e^{-\left(r_{1}-p_{1}\right) \tau_{0}}\right)^{2-\frac{r_{2} \lambda_{2} k_{1}}{r_{1}}}} \int_{0}^{\tau_{0}} e^{r_{3} s} I_{3}(s)\left(\int_{0}^{s} \frac{e^{\left(r_{2}-p_{2}-m\right) u} I_{2}(u)}{e^{r_{3} u} I_{3}(u)} d u\right) d s, \\
& c_{0}^{\prime}=\frac{\lambda_{1} e^{-\left(r_{1}-p_{1}\right) \tau_{0}} T_{1}^{-1} k_{1}\left(r_{1}-p_{1}\right)\left(T_{1}-e^{-\left(r_{1}-p_{1}\right) \tau_{0}}\right)}{\left(1-e^{-\left(r_{1}-p_{1}\right) \tau_{0}}\right)^{2-\frac{r_{3} \lambda_{3} k_{1}}{r_{1}}}} \int_{0}^{\tau_{0}} e^{r_{3} u} I_{3}(u) d u, \\
& e_{0}^{\prime}=1-\left(T_{2}-R\right) e^{\left[r_{2}-m-p_{2}\right] \tau_{0}} T_{1}^{-\frac{r_{2} \lambda_{2} k_{1}}{r_{1}}} e^{-\frac{r_{2} \lambda_{2} k_{1}\left(r_{1}-p_{1}\right)}{r_{1}} \tau_{0}}, \\
& i_{0}^{\prime}=1-T_{3} e^{r_{3} \tau_{0}} T_{1}^{-\frac{r_{3} \lambda_{3} k_{1}}{r_{1}}} e^{-\frac{r_{3} \lambda_{3} k_{1}\left(r_{1}-p_{1}\right)}{r_{1}}} \tau_{0},
\end{aligned}
$$

and

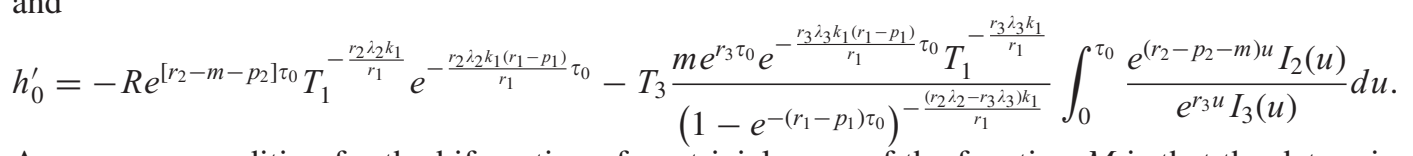

A necessary condition for the bifurcation of nontrivial zeros of the function $M$ is that the determinant of $D_{X} M(0,(0,0,0))$ is equal to zero, i.e. $a_{0}^{\prime} \cdot e_{0}^{\prime} \cdot i_{0}^{\prime}=0$. From stability of $x_{s}$ we have $(10)$, i.e. $a_{0}^{\prime} \neq 0$, it follows that $e_{0}^{\prime} \cdot i_{0}^{\prime}=0$.

We have $e_{0}^{\prime}=0$ for

$$
\tau_{0}=\frac{\ln \left[\left(T_{2}-R\right)^{-1} T_{1}^{\frac{r_{2} \lambda_{2} k_{1}}{r_{1}}}\right]}{r_{2}-m-p_{2}-\frac{r_{2} \lambda_{2} k_{1}\left(r_{1}-p_{1}\right)}{r_{1}}}
$$

and $i_{0}^{\prime}=0$ for

$$
\tau_{0}=\frac{\ln \left[T_{3}^{-1} T_{1}^{\frac{r_{3} \lambda_{3} k_{1}}{r_{1}}}\right]}{r_{3}-\frac{r_{3} \lambda_{3} k_{1}\left(r_{1}-p_{1}\right)}{r_{1}}} .
$$

It remains to find sufficient conditions to obtain bifurcation.

We have the following results.

Theorem 4.1: If conditions (11), (12), (14), (15) and (19) hold, then there exists $\epsilon_{0}>0$, such that for all $\left|\lambda_{2}\right|<\epsilon_{0}$, the problem (1)-(6) has a nontrivial periodic solutions. 


\section{WMLS 2014}

More specifically, there exists $\beta>0$, such that for all $0<\alpha<\beta$, we have a bifurcation of nontrivial $\left(\tau_{0}+\bar{\tau}(\alpha)\right)$-periodic solution with initial condition $\left(x_{0}+\left(\frac{c_{0}^{\prime} h_{0}^{\prime}}{a_{0}^{\prime} i_{0}^{\prime}}-\frac{b_{0}^{\prime}}{a_{0}^{\prime}}\right) \alpha+z_{1}(\bar{\tau}(\alpha), \alpha), \alpha,-\frac{h_{0}^{\prime}}{i_{0}^{\prime}} \alpha+z_{3}(\bar{\tau}(\alpha), \alpha)\right)$ where

$$
\begin{aligned}
& C_{2}\left(\lambda_{2}\right)=-2\left(T_{2}-R\right)\left(\frac{c_{0}^{\prime} h_{0}^{\prime}-b_{0}^{\prime} i_{0}^{\prime}}{a_{0}^{\prime} i_{0}^{\prime}}\right) \lambda_{2} r_{2} e^{\left(r_{2}-p_{2}-m\right) \tau_{0}} e^{-\frac{r_{2} \lambda_{2} k_{1}\left(r_{1}-p_{1}\right)}{r_{1}} \tau_{0}} T_{1}^{-\frac{r_{2} \lambda_{2} k_{1}}{r_{1}}} \\
& \times \int_{0}^{\tau_{0}} e^{\left(r_{1}-p_{1}\right) u}\left(\frac{e^{\left(r_{1}-p_{1}\right) u}\left(T_{1}-e^{-\left(r_{1}-p_{1}\right) \tau_{0}}\right)+1-T_{1}}{1-e^{-\left(r_{1}-p_{1}\right) \tau_{0}}}\right)^{-2} d u \\
& -2 \lambda_{1} k_{1}\left(T_{2}-R\right) \lambda_{2} r_{2} e^{\left(r_{2}-p_{2}-m\right) \tau_{0}} e^{-\frac{r_{2} \lambda_{2} k_{1}\left(r_{1}-p_{1}\right)}{r_{1}}} \tau_{0} T_{1}^{-\frac{r_{2} \lambda_{2} k_{1}}{r_{1}}} \frac{\left(r_{1}-p_{1}\right)\left(T_{1}-e^{-\left(r_{1}-p_{1}\right) \tau_{0}}\right)}{\left(1-e^{-\left(r_{1}-p_{1}\right) \tau_{0}}\right)^{-\frac{r_{2} \lambda_{2} k_{1}}{r_{1}}}} \\
& \times \int_{0}^{\tau_{0}} e^{\left(r_{1}-p_{1}\right) u}\left(e^{\left(r_{1}-p_{1}\right) u}\left(T_{1}-e^{-\left(r_{1}-p_{1}\right) \tau_{0}}\right)+1-T_{1}\right)^{-2}\left(\int_{0}^{u} e^{\left(r_{2}-p_{2}-m\right) s} I_{2}(s) d s\right) d u \\
& +\left(T_{2}-R\right) \frac{2 r_{2}}{k_{2}} e^{\left(r_{2}-p_{2}-m\right) \tau_{0}} e^{-\frac{r_{2} \lambda_{2} k_{1}\left(r_{1}-p_{1}\right)}{r_{1}} \tau_{0}} T_{1}^{-\frac{r_{2} \lambda_{2} k_{1}}{r_{1}}} \\
& \times \int_{0}^{\tau_{0}} e^{\left(r_{2}-p_{2}-m\right) u}\left(\frac{e^{\left(r_{1}-p_{1}\right) u}\left(T_{1}-e^{-\left(r_{1}-p_{1}\right) \tau_{0}}\right)+1-T_{1}}{1-e^{-\left(r_{1}-p_{1}\right) \tau_{0}}}\right)^{-\frac{r_{2} \lambda_{2} k_{1}}{r_{1}}} d u \\
& +2\left(T_{2}-R\right)\left(\frac{2 r_{2}}{k_{2}}+r_{2} \lambda_{2}\right) e^{\left(r_{2}-p_{2}-m\right) \tau_{0}} e^{-\frac{r_{2} \lambda_{2} k_{1}\left(r_{1}-p_{1}\right)}{r_{1}} \tau_{0}} T_{1}^{-\frac{r_{2} \lambda_{2} k_{1}}{r_{1}}} \\
& \times \int_{0}^{\tau_{0}} \frac{m e^{r_{3} r}\left(e^{\left(r_{1}-p_{1}\right) r}\left(T_{1}-e^{-\left(r_{1}-p_{1}\right) \tau_{0}}\right)+1-T_{1}\right)^{-\frac{r_{3} \lambda_{3} k_{1}}{r_{1}}}}{\left(1-e^{-\left(r_{1}-p_{1}\right) \tau_{0}}\right)^{-\frac{r_{2} \lambda_{2} k_{1}}{r_{1}}}}\left(\int_{0}^{r} \frac{e^{\left(r_{2}-p_{2}-m\right) u} I_{2}(u)}{e^{r_{3} u} I_{3}(u)} d u\right) d r \\
& +2 \lambda_{1}\left(T_{2}-R\right) \frac{h_{0}^{\prime}}{i_{0}^{\prime}} \lambda_{2} r_{2} e^{\left(r_{2}-p_{2}-m\right) \tau_{0}} e^{-\frac{r_{2} \lambda_{2} k_{1}\left(r_{1}-p_{1}\right)}{r_{1}}} \tau_{0} T_{1}^{-\frac{r_{2} \lambda_{2} k_{1}}{r_{1}}} \frac{k_{1}\left(r_{1}-p_{1}\right)\left(T_{1}-e^{-\left(r_{1}-p_{1}\right) \tau_{0}}\right)}{\left(1-e^{-\left(r_{1}-p_{1}\right) \tau_{0}}\right)^{2-\frac{r_{3} \lambda_{3} k_{1}}{r_{1}}}} \\
& \times \int_{0}^{\tau_{0}} e^{\left(r_{1}-p_{1}\right) r}\left(\frac{e^{\left(r_{1}-p_{1}\right) r}\left(T_{1}-e^{-\left(r_{1}-p_{1}\right) \tau_{0}}\right)+1-T_{1}}{1-e^{-\left(r_{1}-p_{1}\right) \tau_{0}}}\right)^{-2}\left(\int_{0}^{r} e^{r_{3} u} I_{3}(u) d u\right) d r \\
& -2\left(T_{2}-R\right) \frac{h_{0}^{\prime}}{i_{0}^{\prime}}\left(\frac{2 r_{2}}{k_{2}}+r_{2} \lambda_{2}\right) e^{\left(r_{2}-p_{2}-m\right) \tau_{0}} e^{-\frac{r_{2} \lambda_{2} k_{1}\left(r_{1}-p_{1}\right)}{r_{1}} \tau_{0}} T_{1}^{-\frac{r_{2} \lambda_{2} k_{1}}{r_{1}}} \\
& \times \int_{0}^{\tau_{0}} e^{r_{3} u}\left(\frac{e^{\left(r_{1}-p_{1}\right) u}\left(T_{1}-e^{-\left(r_{1}-p_{1}\right) \tau_{0}}\right)+1-T_{1}}{1-e^{-\left(r_{1}-p_{1}\right) \tau_{0}}}\right)^{-\frac{r_{3} \lambda_{3} k_{1}}{r_{1}}} d u,
\end{aligned}
$$

$$
\begin{aligned}
B_{2}\left(\lambda_{2}\right)= & -\left(T_{2}-R\right)\left(r_{2}-p_{2}-m\right) e^{\left(r_{2}-p_{2}-m\right) \tau_{0}} e^{-\frac{r_{2} \lambda_{2} k_{1}\left(r_{1}-p_{1}\right)}{r_{1}} \tau_{0}} T_{1}^{-\frac{r_{2} \lambda_{2} k_{1}}{r_{1}}} \\
& +\frac{r_{2} \lambda_{2} k_{1}}{r_{1}}\left(T_{2}-R\right) e^{\left(r_{2}-p_{2}-m\right) \tau_{0}}\left(\frac{\left(r_{1}-p_{1}\right)\left(T_{1}-e^{-\left(r_{1}-p_{1}\right) \tau_{0}}\right)}{1-e^{-\left(r_{1}-p_{1}\right) \tau_{0}}}\right) e^{-\frac{r_{2} \lambda_{2} k_{1}\left(r_{1}-p_{1}\right)}{r_{1}}} \tau_{0} T_{1}^{-1-\frac{r_{2} \lambda_{2} k_{1}}{r_{1}}} \\
& +\left(T_{2}-R\right) \frac{k_{1}\left(1-T_{1}\right)\left(r_{1}-p_{1}\right)^{2} e^{-\left(r_{1}-p_{1}\right) \tau_{0}}}{r_{1}} \lambda_{2} r_{2} e^{\left(r_{2}-p_{2}-m\right) \tau_{0}} e^{-\frac{r_{2} \lambda_{2} k_{1}\left(r_{1}-p_{1}\right)}{r_{1}} \tau_{0}} T_{1}^{-\frac{r_{2} \lambda_{2} k_{1}}{r_{1}}} \\
& \times \int_{0}^{\tau_{0}} e^{\left(r_{1}-p_{1}\right) u}\left(e^{\left(r_{1}-p_{1}\right) u}\left(T_{1}-e^{-\left(r_{1}-p_{1}\right) \tau_{0}}\right)+1-T_{1}\right)^{-2} d u
\end{aligned}
$$


$\bar{\tau}(\alpha)=-\frac{C_{2}\left(\lambda_{2}\right)}{2 B_{2}\left(\lambda_{2}\right)} \alpha+o(\alpha)$

$z_{1}(\bar{\tau}(\alpha), \alpha)=\frac{k_{1}\left(1-T_{1}\right)\left(r_{1}-p_{1}\right)^{2} e^{-\left(r_{1}-p_{1}\right) \tau_{0}}}{r_{1}\left(1-e^{-\left(r_{1}-p_{1}\right) \tau_{0}}\right)^{2}} \bar{\tau}+o(\alpha)$

and $z_{3}(\bar{\tau}(\alpha), \alpha)=o(\alpha)$.

Proof: From the condition of the theorem we have $\operatorname{dim} \operatorname{ker}\left(D_{X} M(0,(0,0,0))\right)=1$ and span $\left\{Y_{0}\right\}=\operatorname{ker}\left(D_{X} M(0,(0,0,0))\right)$. Then (17) gives

$$
\left\{\begin{array}{l}
M_{1}\left(\bar{\tau}, \alpha Y_{0}+Z\right)=0, \\
M_{2}\left(\bar{\tau}, \alpha Y_{0}+Z\right)=0, \\
M_{3}\left(\bar{\tau}, \alpha Y_{0}+Z\right)=0,
\end{array}\right.
$$

where $Y_{0}=\left(\frac{c_{0}^{\prime} h_{0}^{\prime}}{a_{0}^{\prime} i_{0}^{\prime}}-\frac{b_{0}^{\prime}}{a_{0}^{\prime}}, 1,-\frac{h_{0}^{\prime}}{i_{0}^{\prime}}\right), Z=\left(z_{1}, 0, z_{3}\right),\left(\alpha, z_{1}, z_{3}\right) \in \mathbb{R}^{3}$ and $X=\alpha Y_{0} \oplus Z$.

From the first and last equations of (21), we see that

$$
\operatorname{det}\left(\begin{array}{ll}
\frac{\partial M_{1}(0,(0,0,0))}{\partial z_{1}} & \frac{\partial M_{1}(0,(0,0,0))}{\partial z_{3}} \\
\frac{\partial M_{3}(0,(0,0,0))}{\partial z_{1}} & \frac{\partial M_{3}(0,(0,0,0))}{\partial z_{3}}
\end{array}\right)=\operatorname{det}\left(\begin{array}{cc}
a_{0} & c_{0} \\
0 & \dot{i}_{0}
\end{array}\right)=\dot{a}_{0} \cdot \dot{i}_{0} \neq 0
$$

Therefore, by the implicit function theorem we can solve the equations $M_{1}\left(\bar{\tau}, \alpha Y_{0}+Z\right)=0$ and $M_{3}\left(\bar{\tau}, \alpha Y_{0}+Z\right)=0$ near $(0,0,0)$ with respect to $Z$ as a function of $\bar{\tau}$ and $\alpha$, and find $Z=Z(\bar{\tau}, \alpha)$ such that $\mathrm{Z}(0,0)=(0,0,0)$,

$$
M_{1}\left(\bar{\tau},\left(\left(\frac{c_{0}^{\prime} h_{0}^{\prime}}{a_{0}^{\prime} i_{0}^{\prime}}-\frac{b_{0}^{\prime}}{a_{0}^{\prime}}\right) \alpha+z_{1}(\bar{\tau}, \alpha), \alpha,-\frac{h_{0}^{\prime}}{i_{0}^{\prime}} \alpha+z_{3}(\bar{\tau}, \alpha)\right)\right)=0
$$

and

$$
M_{3}\left(\bar{\tau},\left(\left(\frac{c_{0}^{\prime} h_{0}^{\prime}}{a_{0}^{\prime} i_{0}^{\prime}}-\frac{b_{0}^{\prime}}{a_{0}^{\prime}}\right) \alpha+z_{1}(\bar{\tau}, \alpha), \alpha,-\frac{h_{0}^{\prime}}{i_{0}^{\prime}} \alpha+z_{3}(\bar{\tau}, \alpha)\right)\right)=0
$$

Moreover, $\frac{\partial z_{1}}{\partial \alpha}(0,0)=\frac{\partial z_{3}}{\partial \bar{\tau}}(0,0)=\frac{\partial z_{3}}{\partial \alpha}(0,0)=0$ and

$$
\frac{\partial z_{1}}{\partial \bar{\tau}}(0,0)=\frac{k_{1}\left(1-T_{1}\right)\left(r_{1}-p_{1}\right)^{2} e^{-\left(r_{1}-p_{1}\right) \tau_{0}}}{r_{1}\left(1-e^{-\left(r_{1}-p_{1}\right) \tau_{0}}\right)^{2}} .
$$

It remains to solve the following equation

$$
f_{2}(\bar{\tau}, \alpha)=M_{2}\left(\bar{\tau},\left(\left(\frac{c_{0}^{\prime} h_{0}^{\prime}}{a_{0}^{\prime} i_{0}^{\prime}}-\frac{b_{0}^{\prime}}{a_{0}^{\prime}}\right) \alpha+z_{1}(\bar{\tau}, \alpha), \alpha,-\frac{h_{0}^{\prime}}{i_{0}^{\prime}} \alpha+z_{3}(\bar{\tau}, \alpha)\right)\right)=0,
$$

it is called a determining equation and the number of its solutions is equal to the number of periodic solutions of (1)-(6) (see [2]). From the Taylor development of $f_{2}$, we have $f_{2}(0,0)=0, \frac{\partial f_{2}(0,0)}{\partial \bar{\tau}}=$ $\frac{\partial f_{2}(0,0)}{\partial \alpha}=0$.

Let $A_{2}=\frac{\partial^{2} f_{2}(0,0)}{\partial \bar{\tau}^{2}}, B_{2}\left(\lambda_{2}\right)=\frac{\partial^{2} f_{2}(0,0)}{\partial \bar{\tau} \partial \alpha}$ and $C_{2}\left(\lambda_{2}\right)=\frac{\partial^{2} f_{2}(0,0)}{\partial \alpha^{2}}$. 
We find $A_{2}=0$. Moreover for $\lambda_{2}=0$ we have $B_{2}(0)=-\left(T_{2}-R\right)\left(r_{2}-p_{2}-m\right) e^{\left(r_{2}-p_{2}-m\right) \tau_{0}}<0$ and

$$
\begin{aligned}
C_{2}(0)= & 2\left(T_{2}-R\right) \frac{r_{2}}{k_{2}} e^{\left(r_{2}-p_{2}-m\right) \tau_{0}}\left(\frac{e^{\left(r_{2}-p_{2}-m\right) \tau_{0}}-1}{r_{2}-p_{2}-m}\right) \\
& +\int_{0}^{\tau_{0}} \frac{4 m \frac{r_{2}}{k_{2}}\left(T_{2}-R\right) e^{\left(r_{2}-p_{2}-m\right) \tau_{0}} e^{r_{3} r} I_{3}(r)}{e^{\left(r_{1}-p_{1}\right) r}\left(T_{1}-e^{-\left(r_{1}-p_{1}\right) \tau_{0}}\right)+1-T_{1}}\left(\int_{0}^{r} \frac{e^{\left(r_{1}-p_{1}\right) u}\left(T_{1}-e^{-\left(r_{1}-p_{1}\right) \tau_{0}}\right)+1-T_{1}}{e^{-\left(r_{2}-r_{3}-p_{2}-m\right) u} I_{3}(u)} d u\right) d r \\
& -4\left(T_{2}-R\right) \frac{h_{0}^{\prime}}{i_{0}^{\prime}} \frac{r_{2}}{k_{2}} \int_{0}^{\tau_{0}} \frac{e^{\left(r_{2}-p_{2}-m\right) \tau_{0}}\left(1-e^{-\left(r_{1}-p_{1}\right) \tau_{0}}\right)^{\frac{r_{3} \lambda_{3} k_{1}}{r_{1}}} e^{r_{3} u} I_{3}(u)}{e^{\left(r_{1}-p_{1}\right) u}\left(T_{1}-e^{-\left(r_{1}-p_{1}\right) \tau_{0}}\right)+1-T_{1}} d u>0
\end{aligned}
$$

since $i_{0}^{\prime}>0$ and $h_{0}^{\prime}<0$.

Hence

$$
f_{2}(\bar{\tau}, \alpha)=B_{2}\left(\lambda_{2}\right) \bar{\tau} \alpha+C_{2}\left(\lambda_{2}\right) \frac{\alpha^{2}}{2}+o\left(|\alpha|^{2}+|\bar{\tau}|^{2}\right)
$$

By taking $\bar{\tau}=\sigma \alpha$, we have $f_{2}(\sigma \alpha, \alpha)=\frac{\alpha^{2}}{2} g_{2}(\sigma, \alpha)$ where $g_{2}(\sigma, \alpha)=2 B_{2}\left(\lambda_{2}\right) \sigma+C_{2}\left(\lambda_{2}\right)+o_{\alpha}\left(1+\sigma^{2}\right)$. Further $\frac{\partial g_{2}}{\partial \sigma}(\sigma, 0)=2 B_{2}\left(\lambda_{2}\right)$ and $g_{2}(\sigma, 0)=2 B_{2}\left(\lambda_{2}\right) \sigma+C_{2}\left(\lambda_{2}\right)$.

So, for $\lambda_{2}$ small enough we have $\sigma_{0}=-\frac{C_{2}\left(\lambda_{2}\right)}{2 B_{2}\left(\lambda_{2}\right)}, g_{2}\left(\sigma_{0}, 0\right)=0$ and $\frac{\partial g_{2}}{\partial \sigma}\left(\sigma_{0}, 0\right) \neq 0$. Using the implicit function theorem we find a function $\sigma(\alpha)$ such that for $\alpha$ and $\lambda_{2}$ small enough $g_{2}(\sigma(\alpha), \alpha)=0$ and $\sigma(0)=\sigma_{0}=-\frac{C_{2}\left(\lambda_{2}\right)}{2 B_{2}\left(\lambda_{2}\right)}>0$.

Then $\bar{\tau}(\alpha)=-\frac{C_{2}\left(\lambda_{2}\right)}{2 B_{2}\left(\lambda_{2}\right)} \alpha+o(\alpha)$ and $f_{2}(\bar{\tau}(\alpha), \alpha)=0$ for $\alpha$ and $\lambda_{2}$ small enough.

We have the following results.

Theorem 4.2: If conditions (11), (13)-(15) and (20) hold, then there exists $\epsilon_{0}>0$, such that for all $\left|\lambda_{3}\right|<\epsilon_{0}$, the problem (1)-(6) has a nontrivial periodic solutions. More specifically, there exists $\beta>0$, such that for all $0<\alpha<\beta$, we have a bifurcation of nontrivial $\left(\tau_{0}+\bar{\tau}(\alpha)\right)$-periodic solution with initial condition $\left(x_{0}-\frac{c_{0}^{\prime}}{a_{0}^{\prime}} \alpha+z_{1}(\bar{\tau}, \alpha), z_{2}(\bar{\tau}, \alpha), \alpha\right)$ where

$$
\begin{aligned}
C_{3}\left(\lambda_{3}\right)= & \frac{c_{0}^{\prime}}{a_{0}^{\prime}} \frac{2 T_{3} e^{r_{3}\left(1-\frac{\lambda_{3} k_{1}\left(r_{1}-p_{1}\right)}{r_{1}}\right) \tau_{0}}}{T_{1}^{\frac{r_{3} \lambda_{3} k_{1}}{r_{1}}}} \int_{0}^{\tau_{0}}-\lambda_{3} r_{3} e^{\left(r_{1}-p_{1}\right) u}\left(\frac{e^{\left(r_{1}-p_{1}\right) u}\left(T_{1}-e^{-\left(r_{1}-p_{1}\right) \tau_{0}}\right)+1-T_{1}}{1-e^{-\left(r_{1}-p_{1}\right) \tau_{0}}}\right)^{-2} d u \\
& -\frac{T_{3} e^{r_{3}\left(1-\frac{r_{3} \lambda_{3} k_{1}\left(r_{1}-p_{1}\right)}{r_{1}}\right) \tau_{0}}}{T_{1}^{\frac{r_{3} \lambda_{3} k_{1}}{r_{1}}}} \int_{0}^{\tau_{0}} 2 \lambda_{3} r_{3} \lambda_{1} e^{\left(r_{1}-p_{1}\right) r}\left(\frac{e^{\left(r_{1}-p_{1}\right) r}\left(T_{1}-e^{-\left(r_{1}-p_{1}\right) \tau_{0}}\right)+1-T_{1}}{1-e^{-\left(r_{1}-p_{1}\right) \tau_{0}}}\right)^{-2} \\
& \times \frac{k_{1}\left(r_{1}-p_{1}\right)\left(T_{1}-e^{-\left(r_{1}-p_{1}\right) \tau_{0}}\right)}{\left(1-e^{-\left(r_{1}-p_{1}\right) \tau_{0}}\right)^{2-\frac{r_{3} \lambda_{3} k_{1}}{r_{1}}}}\left(\int_{0}^{r} e^{r_{3} u}\left(e^{\left(r_{1}-p_{1}\right) u}\left(T_{1}-e^{-\left(r_{1}-p_{1}\right) \tau_{0}}\right)+1-T_{1}\right)^{1-\frac{r_{3} \lambda_{3} k_{1}}{r_{1}}} d u\right) d r \\
& +T_{3} e^{r_{3} \tau_{0}} e^{-\frac{r_{3} \lambda_{3} k_{1}\left(r_{1}-p_{1}\right)}{r_{1}}} \tau_{0} T_{1}^{-\frac{r_{3} \lambda_{3} k_{1}}{r_{1}}} \int_{0}^{\tau_{0}} \frac{2 r_{3}}{k_{3}} e^{r_{3} r}\left(\frac{e^{\left(r_{1}-p_{1}\right) r}\left(T_{1}-e^{\left.-\left(r_{1}-p_{1}\right) \tau_{0}\right)}\right)+1-T_{1}}{1-e^{-\left(r_{1}-p_{1}\right) \tau_{0}}} d r,\right.
\end{aligned}
$$

$$
\begin{aligned}
B_{3}\left(\lambda_{3}\right)= & -T_{3} r_{3} e^{r_{3} \tau_{0}} e^{-\frac{r_{3} \lambda_{3} k_{1}\left(r_{1}-p_{1}\right)}{r_{1}} \tau_{0}} T_{1}^{-\frac{r_{3} \lambda_{3} k_{1}}{r_{1}}} \\
& +\frac{r_{3} \lambda_{3} k_{1}}{r_{1}} T_{3} e^{r_{3} \tau_{0}}\left(\frac{\left(r_{1}-p_{1}\right)\left(T_{1}-e^{-\left(r_{1}-p_{1}\right) \tau_{0}}\right)}{1-e^{-\left(r_{1}-p_{1}\right) \tau_{0}}}\right) e^{-\frac{r_{3} \lambda_{3} k_{1}\left(r_{1}-p_{1}\right)}{r_{1}} \tau_{0}} T_{1}^{-1-\frac{r_{3} \lambda_{3} k_{1}}{r_{1}}}
\end{aligned}
$$




$$
\begin{aligned}
& -T_{3} T_{1} \frac{1}{a_{0}^{\prime}} \frac{k_{1}\left(1-T_{1}\right)\left(r_{1}-p_{1}\right)^{2} e^{-\left(r_{1}-p_{1}\right) \tau_{0}}\left(T_{1}-e^{-\left(r_{1}-p_{1}\right) \tau_{0}}\right)}{r_{1} T_{1}^{2}} e^{r_{3} \tau_{0}} e^{-\frac{r_{3} r_{3} k_{1}\left(r_{1}-p_{1}\right)}{r_{1}} \tau_{0}} T_{1}^{-\frac{r_{2} z_{k} k_{1}}{r_{1}}} \\
& \times \int_{0}^{\tau_{0}}-\lambda_{3} r_{3} e^{\left(r_{1}-p_{1}\right) u}\left(e^{\left(r_{1}-p_{1}\right) u}\left(T_{1}-e^{-\left(r_{1}-p_{1}\right) \tau_{0}}\right)+1-T_{1}\right)^{-2} d u
\end{aligned}
$$

$\bar{\tau}(\alpha)=-\frac{C_{3}\left(\lambda_{3}\right)}{2 B_{3}\left(\lambda_{3}\right)} \alpha+o(\alpha)$

$z_{1}(\bar{\tau}(\alpha), \alpha)=\frac{k_{1}\left(1-T_{1}\right)\left(r_{1}-p_{1}\right)^{2} e^{-\left(r_{1}-p_{1}\right) \tau_{0}}}{r_{1}\left(1-e^{-\left(r_{1}-p_{1}\right) \tau_{0}}\right)^{2}} \bar{\tau}+o(\alpha)$

and $z_{2}(\bar{\tau}(\alpha), \alpha)=o(\alpha)$.

Proof: Using the same argument cited above, we have $\operatorname{dim} \operatorname{ker}\left(D_{X} M(0,(0,0,0))\right)=1$,

$\operatorname{span}\left\{Y_{0}\right\}=\operatorname{ker}\left(D_{X} M(0,(0,0,0))\right)$ and equation (17) is equivalent to $(21)$ where $Y_{0}=\left(-\frac{c_{0}^{\prime}}{a_{0}^{\prime}}, 0,1\right)$, $Z=\left(z_{1}, z_{2}, 0\right)$ and $\left(\alpha, z_{1}, z_{2}\right) \in \mathbb{R}^{3}$.

From the first and second equations of (21), we see that

$$
\operatorname{det}\left(\begin{array}{ll}
\frac{\partial M_{1}(0,(0,0,0))}{\partial z_{1}} & \frac{\partial M_{1}(0,(0,0,0))}{\partial z_{3}} \\
\frac{\partial M_{2}(0,(0,0,0))}{\partial z_{1}} & \frac{\partial M_{2}(0,(0,0,0))}{\partial z_{3}}
\end{array}\right)=\operatorname{det}\left(\begin{array}{cc}
a_{0} & b_{0} \\
0 & \dot{e}_{0}
\end{array}\right)=\dot{a}_{0} \cdot \dot{e}_{0} \neq 0 .
$$

Therefore, by the implicit function theorem, we can solve the equations $M_{1}\left(\bar{\tau}, \alpha Y_{0}+Z\right)=0$ and $M_{2}\left(\bar{\tau}, \alpha Y_{0}+Z\right)=0$ near $(0,0,0)$ with respect to $Z$ as a function of $\bar{\tau}$ and $\alpha$, and find $Z=Z(\bar{\tau}, \alpha)$ such that $Z(0,0)=(0,0,0)$,

$$
M_{2}\left(\bar{\tau},\left(\left(-\frac{c_{0}^{\prime}}{a_{0}^{\prime}}\right) \alpha+z_{1}(\bar{\tau}, \alpha), z_{2}(\bar{\tau}, \alpha), \alpha\right)\right)=0
$$

and

$$
M_{3}\left(\bar{\tau},\left(\left(-\frac{c_{0}^{\prime}}{a_{0}^{\prime}}\right) \alpha+z_{1}(\bar{\tau}, \alpha), z_{2}(\bar{\tau}, \alpha), \alpha\right)\right)=0 .
$$

Moreover, $\frac{\partial z_{1}}{\partial \alpha}(0,0)=\frac{\partial z_{2}}{\partial \bar{\tau}}(0,0)=\frac{\partial z_{2}}{\partial \alpha}(0,0)=0$ and

$$
\frac{\partial z_{1}}{\partial \bar{\tau}}(0,0)=\frac{k_{1}\left(1-T_{1}\right)\left(r_{1}-p_{1}\right)^{2} e^{-\left(r_{1}-p_{1}\right) \tau_{0}}}{r_{1}\left(1-e^{-\left(r_{1}-p_{1}\right) \tau_{0}}\right)^{2}} .
$$

It remains to solve the equation

$$
f_{3}(\bar{\tau}, \alpha)=M_{2}\left(\bar{\tau},\left(\left(-\frac{c_{0}^{\prime}}{a_{0}^{\prime}}\right) \alpha+z_{1}(\bar{\tau}, \alpha), z_{2}(\bar{\tau}, \alpha), \alpha\right)\right)=0 .
$$

From the Taylor development of $f_{3}$, we have $f_{3}(0,0)=0, \frac{\partial f_{3}(0,0)}{\partial \bar{\tau}}=\frac{\partial f_{3}(0,0)}{\partial \alpha}=0$.

Let $A_{3}=\frac{\partial^{2} f_{3}(0,0)}{\partial \bar{\tau}^{2}}, B_{3}\left(\lambda_{3}\right)=\frac{\partial^{2} f_{3}(0,0)}{\partial \bar{\tau} \partial \alpha}$ and $C_{3}\left(\lambda_{3}\right)=\frac{\partial^{2} f_{3}(0,0)}{\partial \alpha^{2}}$. 1) $>0$.

We find $A_{3}=0$, moreover for $\lambda_{3}=0$ we have $B_{3}(0)=-T_{3} r_{3} e^{r_{3} \tau_{0}}<0$ and $C_{3}(0)=\frac{2 T_{3}}{k_{3}} e^{r_{3} \tau_{0}}\left(e^{r_{3} \tau_{0}}-\right.$

Hence

$$
f_{3}(\bar{\tau}, \alpha)=B_{3}\left(\lambda_{3}\right) \bar{\tau} \alpha+C_{3}\left(\lambda_{3}\right) \frac{\alpha^{2}}{2}+o\left(|\alpha|^{2}+|\bar{\tau}|^{2}\right) .
$$

By taking $\bar{\tau}=\sigma \alpha$, we have $f_{3}(\sigma \alpha, \alpha)=\frac{\alpha^{2}}{2} g_{3}(\sigma, \alpha)$ where $g_{3}(\sigma, \alpha)=2 B_{3}\left(\lambda_{3}\right) \sigma+C_{2}\left(\lambda_{3}\right)+o_{\alpha}\left(1+\sigma^{2}\right)$. Further $\frac{\partial g_{3}}{\partial \sigma}(\sigma, 0)=2 B_{3}\left(\lambda_{3}\right)$ and $g_{3}(\sigma, 0)=2 B_{3}\left(\lambda_{3}\right) \sigma+C_{3}\left(\lambda_{3}\right)$. 
For $\lambda_{3}$ small enough we have $\sigma_{0}=-\frac{C_{3}\left(\lambda_{3}\right)}{2 B_{3}\left(\lambda_{3}\right)}, g_{3}\left(\sigma_{0}, 0\right)=0$ and $\frac{\partial g_{3}}{\partial \sigma}\left(\sigma_{0}, 0\right) \neq 0$. Using the implicit function theorem we find a function $\sigma(\alpha)$ such that for $\alpha$ and $\lambda_{3}$ small enough $g_{3}(\sigma(\alpha), \alpha)=0$ and $\sigma(0)=\sigma_{0}=-\frac{C_{3}\left(\lambda_{3}\right)}{2 B_{3}\left(\lambda_{3}\right)}>0$.

Then, $\bar{\tau}(\alpha)=-\frac{C_{3}\left(\lambda_{3}\right)}{2 B_{3}\left(\lambda_{3}\right)} \alpha+o(\alpha)$ and $f_{3}(\bar{\tau}(\alpha), \alpha)=0$, for $\alpha$ and $\lambda_{3}$ small enough.

\section{Concluding remarks}

In this work, we have studied a nonlinear mathematical model describing evolution of cell population constituted by three kinds of cells (normal cells, sensitive tumor cells and resistant tumor cells) under chemotherapeutic treatment by two drugs, the first one $\mathbf{P}$ with continuous effect and the second one $\mathbf{T}$ with instantaneous effects. This model is a generalization of those studied by [1], [5-7] and [10]. We have found sufficient conditions for exponential stability of trivial periodic solutions corresponding to eradication of the tumor, after that we have studied conditions of bifurcation of nontrivial periodic solutions which corresponds to the onset of the tumor. Bifurcation of nontrivial periodic solutions are studied and sufficient condition for bifurcation are found.

\section{References}

[1] A. Boudermine, M. Helal and A. Lakmeche, Bifurcation of non trivial periodic solutions for pulsed chemotherapy model, Journal of Mathematical Sciences and Applications, E- Notes, 2 (2014) 2, 22-44.

[2] S. N. Chow and J. Hale, Methods of bifurcation theory, Springer Verlag 1982.

[3] M. He, Z. Li and F. Chen, Permanence, extinction and global attractivity of the periodic GilpinAyala competition system with impulses, Nonlinear Analysis: Real World Applications, 11 (2010) 1537-1551.

[4] G. Iooss, Bifurcation of maps and applications, Study of mathematics, North Holland 1979.

[5] A. Lakmeche and O. Arino, Nonlinear mathematical model of pulsed-therapy of hetergenous tumor, Nonlinear Anal. Real World Appl., 2 (2001) 455-465.

[6] A. Lakmeche and O. Arino, Bifurcation of nontrivial periodic solutions of impulsive differential equations arising in chemotherapeutic treatment, Dynamics Cont. Discr. Impl. Syst., 7 (2000) $265-287$.

[7] Ah. Lakmeche, M. Helal and A. Lakmeche, Pulsed chemotherapy model, Electronic Journal of Mathematical Analysis and Applications, 2 (2014) 1, 127-148.

[8] U. Ledzewicz, M. Naghnaeian and H. Schattler, Optimal response to chemotherapy of mathematical model of tumor-immune dynamics, Journal of Mathematical Biology, 64 (2012) 557-577.

[9] S. Michelson and J. T. Leith, Unexpected equilibria resulting from differing growth rates of subpopulations within heterogeneous tumors, Math. Biosci., 91 (1988) 119-129.

[10] J. C. Panetta, A mathematical model of periodically pulsed chemotherapy: tumor recurrence and metastasis in a competition environement, Bulletin of mathematical Biology, 58 (1996) 3, 425-447.

[11] L. Wang, L. Chen and J. J. Nieto, The dynamics of an epidemic model for pest control with impulsive effect, Nonlinear Analysis: Real World Applications, 11 (2010) 1374-1386.

[12] H. C. Wei, S. F. Hwang, J. T. Lin and T. J. Chen, The role of initial tumor biomass size in a matematical model of periodically pulsed chemotherapy, Computers and Mathematics with Applications, 61 (2011) 3117-3127. 